\title{
Hypothesis of Conservation of Particle Number
}

\author{
Kozo Aoki \\ Grande_Maison_Hosoyama 110, 5-25-7 Hosoyama, Asao-ku, Kawasaki-shi, Kanagawa, Japan \\ E-mail:kozoaoki@gmail.com \\ Received April 13, 2011; revised May 17, 2011; accepted May 24, 2011
}

\begin{abstract}
As for several nuclear reactions, the electroweak interaction is simply explained by a law of conservation of particle number. We find that the positron and electron consist of the three fundamental particles, $\left\{u, \bar{d}, \bar{v}_{e}\right\}$ and $\left\{\bar{u}, d, v_{e}\right\}$, respectively. Furthermore, the members of the second and third generations quark composites consist of the first generation quark and the neutrino of fundamental particles. The particle and its antiparticle pair(or neutrino and its antineutrino pair) have to be an energy quantum (or a photon). The minimum Higgs boson (called "God particle") might be a neutral pion. The fundamental particles are simply up and down quark, neutrino, muon-neutrino, and those anti-particles.
\end{abstract}

Keywords: Members Of Electron And Positron; Member Of Muon, Members Of $W^{+} / W^{-} / Z^{0} / B$ And Higgs Boson, Members Of Second And Third Generations Quark

\section{Introduction}

The common knowledge has been changed from the cosmological doctrine of the ancient greek Thales's famous belief, "Water constituted the principle of all things". Now we have many brain waves again. We put the new conceptual model for several nuclear reactions in fundamental mathematics (set and identity) and particle physics [1-4].

It is believing that the weak interaction is a $\mathrm{CP}$ violation. However it is based on parity conservation due to a conservation of particle number in this study. The neutron decay, $n \rightarrow p+\bar{e}+\bar{v}_{e}+h v$, is well-known nuclear reaction. With a weak boson for parity conservation, we need to innovate the neutron decay ( $\beta$ decay), the neutron-neutron chain reaction, the proton-proton chain reaction ( $\beta^{+}$decay), the tritium decay, the kaon decay, and the $\Lambda$ decay by [5], by a law of conservation of particle number with the up and down quarks, the neutrinos, the muon-neutrinos, and their anti-particles. The members of electron, positron, and other composites are shown. It was drawn up the declaration of independence of the first generation's up and down quark.

\section{A Paradox}

Why an anti-matter is very low than a matter? To work out it, we have a paradox. The early universe was began from the sea of photons, and the early universe had equal parts of the elementary particle and its anti-particle. The broad or cosmological photon $(\gamma)$ means an electromagnetic wave including gamma-ray. We assume that even now the anti-particle is equal amount of particle based on the idea which a photon consists of particleantiparticle pair. Where is an anti-particle? It leads to a consevation of particle number. This is the reversal idea.

\section{Description of Nuclear Reactions}

\subsection{Description of Neutron Decay}

The neutron decay is shown by the following

$$
\begin{gathered}
n \rightarrow p+e^{-}+\bar{v}_{e}+h v \\
\{u, d, d\} \rightarrow\{u, u, d\}+e^{-}+\bar{v}_{e}+h v
\end{gathered}
$$

The $h v$ indicates a photon $(\gamma)$. Here we insert the variable composite $\mathrm{X}$ into the left side to adjust the numbers of elementary particles on both sides. The initial mysterious composite $\mathrm{X}\{\}$ is an empty set.

$$
\begin{gathered}
n+X \rightarrow P+e^{-}+\bar{v}_{e}+h v \\
\{u, d, d\}+X\{\} \rightarrow\{(u, d, d),(u, \bar{d})\}+e^{-}+\bar{v}_{e}+h v
\end{gathered}
$$

The formulas are based on the law of conservation spin of the quantum, the law of conservation charge, and a law of conservation of particle number; the quantity 
adjustment of particle and anti-particle at the initial and final states. We assume the newly defined composite of proton $\{(u, u, d)(d, \bar{d})\} \rightleftharpoons\{(u, d, d, u, \bar{d})\} \rightleftharpoons\{(u, d, d)$ $(u, \bar{d})\}$, instead of the traditional proton $\{p\},\{u, u, d\}$. The $(d, \bar{d})$ pair is an energy quantum on the analogy of a photon. The $(d, \bar{d})$ rest mass is assumed zero, and the spin is $(1 / 2)+(1 / 2)=1$, since a photon mass is zero. Therefore it is considered as a broad (or an extended) photon. It is feasible assumption since the photon is a pair of neutrino and its anti-neutrino. If the $(d, \bar{d})$ is in $P$, the charged pion $(u, \bar{d})$ can exist in $P$. The $u$ and $\bar{d}$ are added into the composite $\mathrm{X}$ due to adjust the quark of number of both sides.

$$
\{u, d, d\}+X\{u, \bar{d}\} \rightarrow\{(u, d, d),(u, \bar{d})\}+e^{-}+\bar{v}_{e}+h v
$$

But a number of neutrino is not ajust. When we think the electrons is not the fundamental particle, the number of neutrino can be ajust.

The $\bar{v}_{e}$ is added into the composite $\mathrm{X}$ due to adjust the number of both sides.

$$
\{u, d, d\}+X\left\{u, \bar{d}, \bar{v}_{e}\right\} \rightarrow\{(u, d, d),(u, \bar{d})\}+e^{-}+\bar{v}_{e}+h v
$$

The $\bar{u}, d$, and $v_{e}$ are added into the composite $\mathrm{X}$ for the neutrality and complementarity.

$$
\{u, d, d\}+X\left\{u, \bar{u}, d, \bar{d}, v_{e}, \bar{v}_{e}\right\} \rightarrow\{(u, d, d),(u, \bar{d})\}+e^{-}+\bar{v}_{e}+h v
$$

If a kind of photon is $h v$, the $\left\{v_{\mu}, \bar{v}_{\mu}\right\}$ are added into the composite $X$. In this paper, the reactions are including a kind of photon $\left.\left\{v_{\mu}, \bar{v}_{\mu}\right\}\right\}$. The $P$ was shown by $\{(u, d, d),(u, \bar{d})\}$.

$$
\{u, d, d\}+X\left\{u, \bar{u}, d, \bar{d}, v_{e}, \bar{v}_{e}, v_{\mu}, \bar{v}_{\mu}\right\} \rightarrow\{(u, d, d),(u, \bar{d})\}+e^{-}+\bar{v}_{e}+\left\{v_{\mu}, \bar{v}_{\mu}\right\}
$$

The composite $\mathrm{X}$ consists of $\left\{u, \bar{u}, d, \bar{d}, \oplus_{e}, \oplus_{\mu}\right\}$. It is a $Z$ boson $\left(Z^{0}\right)$.

The ' $\oplus$ ' indicates a photon.

The ' $\oplus_{e}$ ' indicates the $\left\{v_{e}, \bar{v}_{e}\right\}$ pair.

The ' $\oplus_{\mu}$ ' indicates the $\left\{v_{\mu}, \bar{v}_{\mu}\right\}$ pair.

The ' $\oplus_{e \mu}$ ' indicates the $\left\{\oplus_{e}, \oplus_{\mu}\right\}$ pair.

The $\left\{u, \bar{d}, \bar{v}_{e}\right\}$ and $\left\{\bar{u}, d, v_{e}\right\}$ components are $e^{+}$ and $e^{-}$, respectively. A series of the neutron decay is as follows. Since the lifetime of $Z^{0}$ is very short, there are $\left\{\pi^{0}\right\}$ and $\left\{\oplus_{e \mu}\right\}$ in the initial state.

$$
\begin{aligned}
& n+\left\{\pi^{0}\right\}+\left\{\oplus_{e \mu}\right\} \rightarrow n+Z^{0} \\
& \rightarrow\{u, d, d\}+\left\{u, \bar{u}, d, \bar{d}, \oplus_{e \mu}\right\} \\
& \rightarrow\left\{(u, d, d),(u, \bar{d}),\left(\bar{u}, d, \oplus_{e}, \oplus_{\mu}\right)\right\} \\
& \rightarrow\{(u, d, d),(u, \bar{d})\}+\left\{\left(\bar{u}, d, v_{e}\right), \bar{v}_{e},\left(\oplus_{\mu}\right)\right\} \\
& \rightarrow\{(u, d, d),(u, \bar{d})\}+\left\{\left(\bar{u}, d, v_{e}\right), \bar{v}_{e}\right\}+\left\{\oplus_{\mu}\right\} \\
& \rightarrow\{(u, d, d),(u, \bar{d})\}+\left\{\bar{u}, d, v_{e}\right\}+\left\{\bar{v}_{e}\right\}+\left\{\oplus_{\mu}\right\} \\
& \rightarrow P+e^{-}+\bar{v}_{e}+\oplus_{\mu}
\end{aligned}
$$

The composite $\left\{\bar{u}, d, v_{e}, \bar{v}_{e}\right\}$ indicates the $W^{-}$boson. The $\left\{\bar{u}, d, v_{e}\right\}+\left\{\bar{v}_{e}\right\}$ is equal to $\left(e^{-}+\bar{v}_{e}\right)$ The composite $\left.\left\{\bar{u}, d, v_{e}\right\}\right\}$ indicates the electron $\left(e^{-}\right)$. We can recognize that the spin of electron is simply explained by the electron of three members of fundamental particles.

\subsection{Description of Neutron-Neutron Chain Reaction}

The following is the initial expression for the neutron- neutron chain reaction.

$$
\begin{gathered}
n+n \rightarrow D+e^{-}+\bar{v}_{e}+h v \\
\{u, d, d\}+\{u, d, d\} \rightarrow\{u, u, d\}\{u, d, d\}+e^{-}+\bar{v}_{e}+h v
\end{gathered}
$$

The deuterium, $D=\{u, u, d\}\{u, d, d\}$, is extended to $\{u, u, d\},\{u, \bar{d}\}\{u, d, d\}$. The nuclear reaction is rewritten with $\pi^{0}$, photons, and $Z^{0}$.

$$
\begin{aligned}
& n+n+\left\{\pi^{0}\right\}+\left\{\oplus_{e \mu}\right\} \rightarrow n+n+Z^{0} \\
& \rightarrow\{u, d, d\}+\{u, d, d\}+\left\{u, \bar{u}, d, \bar{d}, \oplus_{e \mu}\right\} \\
& \rightarrow\left\{(u, d, d),(u, d, d),\left(u, \bar{u}, d, \bar{d}, \oplus_{e \mu}\right)\right\} \\
& \rightarrow\{(u, d, d),(u, \bar{d})\}\{u, d, d\}\left\{\bar{u}, d, \oplus_{e}, \oplus_{\mu}\right\} \\
& \rightarrow\{(u, d, d),(u, \bar{d})\}\{u, d, d\}+\left\{\left(\bar{u}, d, v_{e}\right), \bar{v}_{e}, \oplus_{\mu}\right\} \\
& \rightarrow\{(u, d, d),(u, \bar{d})\}\{u, d, d\}+\left\{\left(\bar{u}, d, v_{e}\right), \bar{v}_{e}\right\}+\left\{\oplus_{\mu}\right\} \\
& \rightarrow D+e^{-}+\bar{v}_{e}+\oplus_{\mu}
\end{aligned}
$$

\subsection{Description of Proton-Proton Chain Reaction}

The two proton-proton chain reactions are shown.

$$
\begin{gathered}
p+p \rightarrow D+e^{+}+v_{e}+h v \\
\{u, u, d\}+\{u, u, d\} \rightarrow\{u, u, d\}\{u, d, d\}+e^{+}+v_{e}+h v
\end{gathered}
$$

and

$$
\begin{aligned}
p+p & \rightarrow D+\mu^{+}+v_{\mu} \\
& \rightarrow D+\left(e^{+}+v_{e}+\bar{v}_{\mu}\right)+v_{\mu}
\end{aligned}
$$




$$
\begin{aligned}
& \{u, u, d\}+\{u, u, d\} \rightarrow\{u, u, d\}\{u, d, d\}+\mu^{+}+v_{\mu} \\
& \rightarrow\{u, u, d\}\{u, d, d\}+\left(e^{+}+v_{e}+\bar{v}_{\mu}\right)+v_{\mu}
\end{aligned}
$$

Those are shown with the expression of newly defined proton $\{(u, d, d),(u, \underline{d})\}$.

$$
\begin{aligned}
& P+P+\left\{\oplus_{e \mu}\right\} \\
& \rightarrow\{(u, d, d),(u, \bar{d})\}+\{(u, d, d),(u, \bar{d})\}+\left\{\oplus_{e \mu}\right\} \\
& \rightarrow\{(u, d, d),(u, \bar{d})\}\left\{(u, d, d),(u, \bar{d}),\left(\oplus_{e \mu}\right)\right\} \\
& \rightarrow\{(u, d, d),(u, \bar{d})\}\{u, d, d\}\left\{\left(u, \bar{d}, \oplus_{e}\right), \oplus_{\mu}\right\} \\
& \rightarrow\{(u, d, d),(u, \bar{d})\}\{u, d, d\}+\left\{\left(u, \bar{d}, \bar{v}_{e}\right), v_{e}\right\}+\left\{\oplus_{\mu}\right\} \\
& \rightarrow\{(u, d, d),(u, \bar{d})\}\{u, d, d\}+\left\{u, \bar{d}, \bar{v}_{e}\right\}+\left\{v_{e}\right\}+\left\{\oplus_{\mu}\right\} \\
& \rightarrow D+e^{+}+v_{e}+\oplus_{\mu}
\end{aligned}
$$

and

$$
\begin{aligned}
& P+P+\left\{\oplus_{e \mu}\right\} \\
& \rightarrow\{(u, d, d),(u, \bar{d})\}+\{(u, d, d),(u, \bar{d})\}+\left\{\oplus_{e \mu}\right\} \\
& \rightarrow\{(u, d, d),(u, \bar{d})\}\left\{(u, d, d),(u, \bar{d}),\left(\oplus_{e \mu}\right)\right\} \\
& \rightarrow\{(u, d, d),(u, \bar{d})\}\{u, d, d\}\left\{u, \bar{d}, \oplus_{e}, \oplus_{\mu}\right\} \\
& \rightarrow\{(u, d, d),(u, \bar{d})\}\{u, d, d\}+\left\{u, \bar{d}, v_{e}, \bar{v}_{e}, \bar{v}_{\mu}\right\}+\left\{v_{\mu}\right\} \\
& \rightarrow D+v_{\mu}^{+}+v_{\mu} \\
& \rightarrow\{(u, d, d),(u, \bar{d})\}\{u, d, d\}+\left\{\left(u, \bar{d}, \bar{v}_{e}\right), v_{e}, \bar{v}_{\mu}\right\}+\left\{v_{\mu}\right\} \\
& \rightarrow D+\left(e^{+}+v_{e}+\bar{v}_{\mu}\right)+v_{\mu}
\end{aligned}
$$

The energy quantum, $\left\{\oplus_{\mu}\right\}$, was written in the former reaction. It is unknown whether the $\left\{\oplus_{\mu}\right\}$ can be omit or not. The composite $\left\{u, \bar{d}, v_{e}, \bar{v}_{e}\right\}$ indicates the $W^{+}$boson. This reaction does not occur unless there are the neutrino-antineutrino pairs. The $\left\{u, d, \bar{v}_{e}\right\}$ $\left\{v_{e}\right\}$ is equal to $\left(e^{+}+v_{e}\right)$. The composite $\left\{u, d, \bar{v}_{e}\right\}$ indicates the positron $\left(e^{+}\right)$. The composite $\left\{\bar{u}, d, v_{e}\right.$, $\left.\bar{v}_{e}, \bar{v}_{u}\right\}$ indicates a $\mu^{+}$muon.

The cases with the $Z^{0}$ boson are also considered. All bosons are composites.

$$
\begin{aligned}
& P+P+\left\{\pi^{0}\right\}+\left\{\oplus_{e \mu}\right\} \rightarrow P+P+Z^{0} \\
& \rightarrow\{(u, d, d),(u, \bar{d})\}+\{(u, d, d),(u, \bar{d})\}+\left\{Z^{0}\right\} \\
& \left.\rightarrow\{(u, d, d),(u, \bar{d})\}\left\{(u, d, d),(u, \bar{d}),\left(\pi^{0}\right)\right), \oplus_{e}, \oplus_{\mu}\right\} \\
& \rightarrow\{(u, d, d),(u, \bar{d})\}\{u, d, d\}+\left\{\left(u, \bar{d}, \oplus_{e}\right),\left(\pi^{0}\right), \oplus_{\mu}\right\} \\
& \rightarrow\{(u, d, d),(u, \bar{d})\}\{u, d, d\}+\left\{u, \bar{d}, \bar{v}_{e}\right\}+\left\{v_{e},\left(\pi^{0}\right),\left(\oplus_{\mu}\right)\right\} \\
& \rightarrow D+e^{+}+v_{e}+\left\{\pi^{0}\right\}+\left\{\oplus_{\mu}\right\}
\end{aligned}
$$

and

$$
\begin{aligned}
& P+P+\left\{\pi^{0}\right\}+\left\{\oplus_{e \mu}\right\} \rightarrow P+P+Z^{0} \\
\rightarrow & \{(u, d, d),(u, \bar{d})\}+\{(u, d, d),(u, \bar{d})\}+\left\{Z^{0}\right\} \\
\rightarrow & \{(u, d, d),(u, \bar{d})\}\left\{(u, d, d),(u, \bar{d}),\left(\pi^{0}\right), \oplus_{e}, \oplus_{\mu}\right\} \\
\rightarrow & \{(u, d, d),(u, \bar{d})\}\{u, d, d\}\left\{\left(u, \bar{d}, \oplus_{e \mu}\right),\left(\pi^{0}\right)\right\} \\
\rightarrow & \{(u, d, d),(u, \bar{d})\}\{u, d, d\} \\
& +\left(u, \bar{d}, v_{e}, \bar{v}_{e}, \bar{v}_{\mu}\right)+\left\{v_{\mu}\right\}+\left\{\pi^{0}\right\} \\
& \left.\rightarrow D+v_{\mu}^{+}+v_{\mu}+\left\{\pi^{0}\right\}\right) \\
& \rightarrow\{(u, d, d),(u, \bar{d})\}\{u, d, d\} \\
& +\left\{\left(u, \bar{d}, \bar{v}_{e}\right), v_{e}, \bar{v}_{\mu}\right\}+\left\{v_{\mu}\right\}+\left\{\pi^{0}\right\} \\
& \rightarrow\{(u, d, d),(u, \bar{d})\}\{u, d, d\} \\
& +\left\{\left(u, \bar{d}, \bar{v}_{e}\right), v_{e}, \bar{v}_{\mu}\right\}+\left\{v_{\mu}\right\}+\left\{\pi^{0}\right\}
\end{aligned}
$$

\subsection{Description of Tritium Decay}

The tritium decay is $T \rightarrow^{3} \mathrm{He}^{+}+e^{-}+\bar{v}_{e}+h v$.

$$
\begin{aligned}
T & \rightarrow\{(u, d, d),(u, \bar{d})\}\left\{e^{-}\right\}\{u, d, d\}\{u, d, d\} \\
\rightarrow & \{(u, d, d),(u, \bar{d})\}\left\{e^{-}\right\}\{u, d, d\}\{(u, d, d),(u, \bar{d})\} \\
& +\left\{\bar{u}, d, v_{e}\right\}+\left\{\bar{v}_{e}\right\}+h v \\
& \rightarrow{ }^{3} H e^{+}+e^{-}+\bar{v}_{e}+h v
\end{aligned}
$$

It seems that the $Z^{0}$ boson is missing. The new expression is $T+Z^{0} \rightarrow^{3} \mathrm{He}^{+}+e^{-}+\bar{v}_{e}+h v$

$$
\begin{aligned}
T & +\left(\pi^{0}\right)+\left\{\oplus_{e \mu}\right\} \rightarrow T+Z^{0} \\
\rightarrow & \{(u, d, d),(u, \bar{d})\}\left\{\left(e^{-}\right)\right\}\{u, d, d\}\{u, d, d\} \\
& +\left\{u, \bar{u}, d, \bar{d}, \oplus_{e \mu}\right\} \\
\rightarrow & \{(P)\}\left\{\left(e^{-}\right)\right\}\{(n)\}\{(n)\}+\left\{u, \bar{u}, d, \bar{d}, \oplus_{e}, \oplus_{\mu}\right\} \\
\rightarrow & \{(P)\}\left\{\left(e^{-}\right)\right\}\{(n)\}\{(P)\}\left\{\left(\bar{u}, d, v_{e}, \bar{v}_{e}\right),\left(\oplus_{\mu}\right)\right\} \\
\rightarrow & \{(P)\}\left\{\left(e^{-}\right)\right\}\{(n)\}\{(P)\} \\
& +\left\{\left(\bar{u}, d, v_{e}\right), \bar{v}_{e}\right\}+\left\{\oplus_{\mu}\right\} \\
\rightarrow & \{(P)\}\left\{\left(e^{-}\right)\right\}\{(n)\}\{(P)\} \\
& +\left\{\bar{u}, d, v_{e}\right\}+\left\{\bar{v}_{e}\right\}+\left\{\oplus_{\mu}\right\} \\
\rightarrow & H e^{+}+e^{-}+\bar{v}_{e}+\oplus_{\mu}
\end{aligned}
$$

The $\left\{\left(e^{-}\right)\right\}$is the tritium or Helium's extranuclear electron. 


\section{Identity of Fundamental Particle Composites}

It is considered that the traditional fundamental partricles (a boson, a meson, and a second and third generation's quark) will be a composites, since the electron are not the fundamental particle in this study.

\subsection{Identity of the $Z^{0}$ boson in Early Universe}

In early universe, the relationship among the $Z^{0}$ boson, elementary particles, leptons, and photons is described as follows. In the initial early universe, it is thought that only the photons $\left(\oplus_{u}, \oplus_{d}, \oplus_{e}\right.$, and $\left.\oplus_{\mu}\right)$ exist.

$$
\begin{aligned}
& {\left[\oplus_{u}\right]\left[\oplus_{d}\right]\left[\oplus_{e}\right]\left[\oplus_{\mu}\right]=\left[u, \bar{u}, d, \bar{d}, v_{e}, \bar{v}_{e}, v_{\mu}, \bar{v}_{\mu}\right] \equiv\left[Z^{0}\right]} \\
& =\left[u, \bar{d}, v_{e}, \bar{v}_{e}, v_{\mu}, \bar{v}_{\mu}\right][\bar{u}, d] \equiv\left[W^{+}\right]\left[\pi^{-}\right]=[u, \bar{d}]\left[\bar{u}, d, v_{e}, \bar{v}_{e}, v_{\mu}, \bar{v}_{\mu}\right] \equiv\left[\pi^{+}\right]\left[W^{-}\right] \\
& =\left[u, \bar{d}, v_{e}, \bar{v}_{e}, \bar{v}_{\mu}\right][\bar{u}, d]\left[v_{\mu}\right] \equiv\left[\mu^{+}\right]\left[\pi^{-}\right]\left[v_{\mu}\right]=[u, \bar{d}]\left[\bar{u}, d, v_{e}, \bar{v}_{e}, v_{\mu}\right]\left[\bar{v}_{\mu}\right] \equiv\left[\pi^{+}\right]\left[\mu^{-}\right]\left[\bar{v}_{\mu}\right] \\
& =\left[u, \bar{d}, \bar{v}_{e}\right]\left[\bar{u}, d, v_{e}\right]\left[v_{\mu}, \bar{v}_{\mu}\right] \equiv\left[e^{+}\right]\left[e^{-}\right]\left[v_{\mu}, \bar{v}_{\mu}\right]=[u, \bar{d}]\left[\bar{u}, d, v_{e}\right]\left[\bar{v}_{e}\right]\left[v_{\mu}, \bar{v}_{\mu}\right] \equiv\left[\pi^{+}\right]\left[e^{-}\right]\left[\bar{v}_{e}\right]\left[v_{\mu}, \bar{v}_{\mu}\right] \\
& =\left[u, \bar{d}, \bar{v}_{e}\right][\bar{u}, d]\left[v_{e}\right]\left[v_{\mu}, \bar{v}_{\mu}\right] \equiv\left[e^{+}\right]\left[\pi^{-}\right]\left[v_{e}\right]\left[v_{\mu}, \bar{v}_{\mu}\right]=[u, \bar{d}][\bar{u}, d]\left[v_{e}, \bar{v}_{e}\right]\left[v_{\mu}, \bar{v}_{\mu}\right] \equiv\left[\pi^{+}\right]\left[\pi^{-}\right]\left[v_{e}, \bar{v}_{e}\right]\left[v_{\mu}, \bar{v}_{\mu}\right] \\
& =[u, \bar{d}, \bar{u}, d]\left[v_{e}, \bar{v}_{e}\right]\left[v_{\mu}, \bar{v}_{\mu}\right] \equiv\left[\pi^{0}\right]\left[v_{e}, \bar{v}_{e}\right]\left[v_{\mu}, \bar{v}_{\mu}\right]
\end{aligned}
$$

Next is the relationship among elementary particles, protons, neutrons, and photons.

$$
\begin{aligned}
& {\left[\oplus_{u}\right]\left[\oplus_{d}\right]\left[\oplus_{d}\right]\left[\oplus_{u}\right]\left[\oplus_{d}\right]=[u, \bar{u}][d, \bar{d}][d, \bar{d}][u, \bar{u}][d, \bar{d}]} \\
& =[u, d, d][\bar{u}, \bar{d}, \bar{d}][u, \bar{u}, d, \bar{d}] \equiv[n][\bar{n}]\left[\pi^{0}\right] \\
& =[(u, d, d),(u, \bar{d})][(\bar{u}, \bar{d}, \bar{d})(\bar{u}, d)] \equiv\left[(n)\left(\pi^{+}\right)\right]\left[(\bar{n})\left(\pi^{-}\right)\right] \equiv[P][\bar{P}] \\
& =[(u, u, d)(d, \bar{d})][(\bar{u}, \bar{u}, \bar{d})(\bar{d}, d)] \equiv[(p)(d, \bar{d})][(\bar{p})(\bar{d}, d)] \equiv[P][\bar{P}] \\
& =[(u, d, d),(u, \bar{d})][(\bar{u}, \bar{d}, \bar{d})(u, \bar{d})] \equiv\left[(n)\left(\pi^{-}\right)\right]\left[(\bar{n})\left(\pi^{+}\right)\right] \\
& =[(d, d, d)(u, \bar{u})][(\bar{d}, \bar{d}, \bar{d})(u, \bar{u})] \equiv\left[\left(p_{2}\right)(u, \bar{u})\right]\left[\left(\bar{p}_{2}\right)(u, \bar{u})\right] \equiv\left[P_{2}^{-}\right]\left[P_{2}^{+}\right] \\
& =[(u, d, d)(\bar{u}, u)][(\bar{u}, \bar{d}, \bar{d})(d, \bar{d})] \equiv[(n)(\bar{u}, u)][(\bar{n})(d, \bar{d})] \\
& =[(u, u, d)(\bar{u}, d)][(\bar{d}, \bar{d}, \bar{d})(\bar{u}, d)] \equiv\left[(p)\left(\pi^{-}\right)\right]\left[\left(\bar{p}_{2}\right)\left(\pi^{-}\right)\right] \\
& =[(u, d, d)(\bar{d}, d)][(\bar{u}, \bar{d}, \bar{d})(u, \bar{u})] \equiv[(n)(\bar{d}, d)][(\bar{n})(u, \bar{u})] \\
& =[(d, d, d)(u, \bar{d})][(\bar{u}, \bar{u}, \bar{d})(u, \bar{d})] \equiv\left[\left(p_{2}\right)\left(\pi^{+}\right)\right]\left[(\bar{p})\left(\pi^{+}\right)\right]
\end{aligned}
$$

The $P_{2}^{-}$indicates a second newly defined proton with negative charge.

The $P_{2}^{+}$indicates a second newly defined proton with positive charge.

We have the sense of the duality principle between the particle and wave for a photon. The next expression with the $\pi^{0}$ boson is the electron-positron pair annihi- lation by [6]. The gamma-ray $(\gamma)$ indicates the neutrino and its antineutrino connection.

$$
\begin{aligned}
& e^{+}+e^{-} \rightarrow\left\{\left(u, \bar{d}, \bar{v}_{e}\right),\left(\bar{u}, d, v_{e}\right)\right\} \\
& \rightarrow\left\{(u, \bar{d}, \bar{u}, d),\left(v_{e}, \bar{v}_{e}\right)\right\} \rightarrow\left\{\left(\pi^{0}\right),\left(\oplus_{e}\right)\right\} \\
& \rightarrow\left\{\pi^{0}\right\}+\gamma \rightarrow\left\{\pi^{0}\right\}+\left\{\oplus_{e}\right\}
\end{aligned}
$$

$$
\begin{aligned}
& e^{+}+e^{-}+\gamma \rightarrow\left\{\left(u, \bar{d}, \bar{v}_{e}\right),\left(\bar{u}, d, v_{e}\right),\left(v_{\mu}, \bar{v}_{\mu}\right)\right\} \\
& \rightarrow\left\{(u, \bar{d}, \bar{u}, d),\left(v_{e}, \bar{v}_{e}\right),\left(v_{\mu}, \bar{v}_{\mu}\right)\right\} \\
& \rightarrow\left\{\left(\pi^{0}\right), \oplus_{e}, \oplus_{\mu}\right\} \rightarrow\left\{\pi^{0}\right\}+\left\{\oplus_{e}\right\}+\left\{\oplus_{\mu}\right\} \\
& \rightarrow\left\{\pi^{0}\right\}+2 \gamma
\end{aligned}
$$

Next indicates an electron-positron pair creation.

$$
\begin{aligned}
& \pi^{0}+\gamma \rightarrow\left\{\pi^{0}, \oplus_{e}\right\} \\
& \rightarrow\left\{\left(u, \bar{d}, \bar{v}_{e}\right),\left(\bar{u}, d, v_{e}\right)\right\} \\
& \rightarrow\left\{u, \bar{d}, \bar{v}_{e}\right\}+\left\{\bar{u}, d, v_{e}\right\} \\
& \rightarrow e^{+}+e^{-}
\end{aligned}
$$


The energy quanta (or the photon) $\left(\left\{\oplus_{u}\right\},\left\{\oplus_{d}\right\}\right.$, $\left\{\oplus_{e}\right\}$ and $\left.\left\{\oplus_{u}\right\}\right)$ indicate $\{u, \bar{u}\},\{d, \bar{d}\},\left\{v_{e}, \bar{v}_{e}\right\}$, and $\left\{v_{\mu}, \bar{v}_{\mu}\right\}$, respectively.

The energy quanta and particle, $\left\{\oplus_{u}\right\},\left\{\oplus_{d}\right\},\left\{\oplus_{e}\right\}$, $\left\{\oplus_{u}\right\}$ and $\left\{\pi^{0}\right\}$ may be dark energy and dark matter in space.

\subsection{Identity of the Strange and Charm quark}

The charged kaons, $K^{+}$and $K^{-}$, consist of $\{u,(\bar{s})\}$ and $\{\bar{u},(s)\}$, respectively. The main $K^{+}$decay is $K^{+}$ $\rightarrow \mu^{+}+v_{\mu}$. The $\mu^{+}$muon consists of $\left\{u, \bar{d}, v_{e}, \bar{v}_{e}, \bar{v}_{\mu}\right\}$.

$$
\begin{aligned}
& K^{+} \rightarrow\left\{\left(u, \bar{d}, v_{e}, \bar{v}_{e}, \bar{v}_{\mu}\right), v_{\mu}\right\}=\left\{u, \bar{d}, \oplus_{e \mu}\right\} \rightarrow\left\{u, \bar{d}, v_{e}, \bar{v}_{e}, \bar{v}_{\mu}\right\}+v_{\mu} \\
& \rightarrow \mu^{+}+v_{\mu} \\
&\left(K^{+}\right)=\{u,(\bar{s})\} \\
&=\left\{u,\left(\bar{d}, \oplus_{e \mu}\right)\right\} \\
&=\left\{(u, \bar{d}), \oplus_{e \mu}\right\} \\
&=\left\{\left(\pi^{+}\right), \oplus_{e \mu}\right\} \\
&(\bar{s})=\left\{\bar{d}, \oplus_{e \mu}\right\}=\left\{\bar{d}, \oplus_{e}, \oplus_{\mu}\right\}
\end{aligned}
$$

In the same way, the $K^{-}$kaon and $s$ quark compositions are found.

$$
\begin{aligned}
\left(K^{-}\right) & =\{\bar{u},(s)\} \\
& =\left\{\bar{u},\left(d, \oplus_{e \mu}\right)\right\} \\
& =\left\{(\bar{u}, d), \oplus_{e \mu}\right\} \\
& =\left\{\left(\pi^{-}\right), \oplus_{e \mu}\right\} \\
(s) & =\left\{d, \oplus_{e \mu}\right\}=\left\{d, \oplus_{e}, \oplus_{\mu}\right\}
\end{aligned}
$$

The charged kaons consist of the charged pion and the neutrino-antineutrino pairs (or energy quanta). The neutral kaons, $K^{0}$ and $\bar{K}^{0}$, consist of $(d, \bar{s})$ and $(\bar{d}, s)$, respectively.

$$
\begin{aligned}
\left(K^{0}\right) & =\{d,(\bar{s})\} \\
& =\left\{d, \bar{d}, \oplus_{e \mu}\right\} \\
& =\left\{\oplus_{d}, \oplus_{e \mu}\right\} \\
\left(\bar{K}^{0}\right) & =\{\bar{d},(s)\} \\
& =\left\{\bar{d}, d, \oplus_{e \mu}\right\} \\
& =\left\{\oplus_{d}, \oplus_{e \mu}\right\} \\
& =\left\{K^{0}\right\}
\end{aligned}
$$

$$
\begin{aligned}
\left(Z^{0}\right) & =\left\{\left(\pi^{0}\right), \oplus_{e \mu}\right\}=\left\{\left(\pi^{0}\right), \oplus_{e}, \oplus_{\mu}\right\} \\
& =\left\{\left(\pi^{+}\right),\left(\pi^{-}\right), \oplus_{e \mu}\right\} \\
& =\left\{(u, \bar{d}),(\bar{u}, d), \oplus_{e \mu}\right\} \\
& =\left\{(u, \bar{u}, d, \bar{d}), \oplus_{e \mu}\right\} \\
& =\left\{(u, \bar{u}),(d, \bar{d}), \oplus_{e \mu}\right\} \\
& =\left\{\oplus_{u}, \oplus_{d}, \oplus_{e \mu}\right\} \\
& =\left\{\oplus_{u},\left(K^{0}\right)\right\} \\
& =\left\{\oplus_{u},\left(\bar{K}^{0}\right)\right\}
\end{aligned}
$$

By the result of the strange quark, the charm and anti-charm quarks are shown from the $\Omega^{0}$ decay by [7].

$$
\begin{aligned}
& \Omega^{0} \rightarrow \Omega^{-}+\pi^{+} \\
\{s, s, c\} \rightarrow\{s, s, s\}+\{u, \bar{d}\} & (c)=\{(s), u, \bar{d}\}=\left\{(s),\left(\pi^{+}\right)\right\} \\
= & \left\{d, \oplus_{e \mu}, u, \bar{d}\right\} \\
= & \left\{d, \oplus_{e \mu},\left(\pi^{+}\right)\right\}=\left\{d,\left(K^{+}\right)\right\} \\
= & \left\{u, d, \bar{d}, \oplus_{e \mu}\right\}=\left\{u,\left(K^{0}\right)\right\} \\
(\bar{c})= & \{(\bar{s}), \bar{u}, d\}=\left\{(\bar{s}),\left(\pi^{-}\right)\right\} \\
= & \left\{\bar{d}, \oplus_{e \mu}, \bar{u}, d\right\} \\
= & \left\{\bar{d}, \oplus_{e \mu},\left(\pi^{-}\right)\right\}=\left\{\bar{d},\left(K^{-}\right)\right\} \\
= & \left\{\bar{u}, d, \bar{d}, \oplus_{e \mu}\right\}=\left\{\bar{u},\left(K^{0}\right)\right\}
\end{aligned}
$$

The second, third, and fourth $K^{+}$decays are shown by $K^{+} \rightarrow \pi^{+}+\pi^{0}, K^{+} \rightarrow \pi^{+}+\pi^{+}+\pi^{-}$, and $K^{+} \rightarrow$ $\pi^{+}+\pi^{0}+\pi^{0}$. It seems that the $Z^{0}$ bosons are missing in the left sides. We consider about $K^{+}+Z^{0} \rightarrow \pi^{+}+\pi^{0}$, $K^{+}+Z^{0} \rightarrow \pi^{+}+\pi^{+}+\pi^{-}$, and $K^{+}+Z^{0}+Z^{0} \rightarrow \pi^{+}+\pi^{0}+\pi^{0}$. Their reactions are revolved.

$$
\begin{aligned}
K^{+}+Z^{0} & \rightarrow\left\{u, \bar{d}, \oplus_{e \mu}\right\}+\left\{u, \bar{u}, d, \bar{d}, \oplus_{e \mu}\right\} \\
& \rightarrow\left\{(u, \bar{d}),(u, \bar{d}, \bar{u}, d), \oplus_{e \mu}, \oplus_{e \mu}\right\} \\
& \rightarrow \pi^{+}+\pi^{0}+\left\{\oplus_{e \mu}, \oplus_{e \mu}\right\} \\
K^{+}+Z^{0} & \rightarrow\left\{u, \bar{d}, \oplus_{e \mu}\right\}+\left\{u, \bar{u}, d, \bar{d}, \oplus_{e \mu}\right\} \\
\rightarrow & \left\{(u, \bar{d}),(u, \bar{d}, \bar{u}, d), \oplus_{e \mu}, \oplus_{e \mu}\right\} \\
& \rightarrow\left\{(u, \bar{d}),(u, \bar{d}),(\bar{u}, d), \oplus_{e \mu}, \oplus_{e \mu}\right\} \\
& \rightarrow \pi^{+}+\pi^{+}+\pi^{-}+\left\{\oplus_{e \mu}, \oplus_{e \mu}\right\}
\end{aligned}
$$


and

$$
\begin{aligned}
& K^{+}+Z^{0}+Z^{0} \\
& \rightarrow\left\{u, \bar{d}, \oplus_{e \mu}\right\}+\left\{u, \bar{u}, d, \bar{d}, \oplus_{e \mu}\right\}+\left\{u, \bar{u}, d, \bar{d}, \oplus_{e \mu}\right\} \\
& \rightarrow\left\{(u, \bar{d}),(u, \bar{d}, \bar{u}, d),(u, \bar{d}, \bar{u}, d),\left(\oplus_{e}, \oplus_{\mu}\right), \oplus_{e \mu}, \oplus_{e \mu}\right\} \\
& \rightarrow \pi^{+}+\pi^{0}+\pi^{0}+\left\{\oplus_{e \mu}, \oplus_{e \mu}, \oplus_{e \mu}\right\}
\end{aligned}
$$

The rare $K^{+}$decay is $K^{+} \rightarrow \pi^{0}+e^{+}+v_{e}$. It seems that the $Z^{0}$ boson is also missing in the left side. We consider about $K^{+}+Z^{0} \rightarrow \pi^{0}+e^{+}+v_{e}$

$$
\begin{aligned}
& K^{+}+Z^{0} \rightarrow\left\{u, \bar{d}, \oplus_{e \mu}\right\}+\left\{u, \bar{u}, d, \bar{d}, v_{e}, \bar{v}_{e}, \oplus_{\mu}\right\} \\
& \rightarrow\left\{(u, \bar{d}, \bar{u}, d),\left(u, \bar{d}, \bar{v}_{e}\right), v_{e}, \oplus_{\mu}, \oplus_{e \mu}\right\} \\
& \rightarrow\{u, \bar{d}, \bar{u}, d\}+\left\{u, \bar{d}, \bar{v}_{e}\right\}+\left\{v_{e}\right\}+\left\{\oplus_{\mu}, \oplus_{e \mu}\right\} \\
& \rightarrow \pi^{0}+e^{+}+v_{e}+\left\{\oplus_{\mu}, \oplus_{e \mu}\right\}
\end{aligned}
$$

It is considered that the $\pi^{+}$creation is more favorable than the $\left(e^{+}+v_{e}\right)$ one by comparing the second, third, and fourth $K^{+}$decays with the rare $K^{+}$one. The $K_{s}$ consists of the $\{d, \bar{s}, s, \bar{d}\}$.

$$
\left(K_{s}\right)=\{d,(\bar{s}),(s), \bar{d}\}=\left\{d,\left(\bar{d}, \oplus_{e \mu}\right),\left(d, \oplus_{e \mu}\right), \bar{d}\right\}
$$

The $K_{s}$ decays are $K_{s} \rightarrow \pi^{+}+\pi^{-}$and $K_{s} \rightarrow$ $\pi^{0}+\pi^{0}$. It seems that the $Z^{0}$ bosons are missing in the left sides. Their reactions are as follows.

$$
\begin{aligned}
& K_{s}+Z^{0} \rightarrow\left\{d,\left(\bar{d}, \oplus_{e \mu}\right),\left(d, \oplus_{e \mu}\right), \bar{d}\right\}+\left\{u, \bar{u}, d, \bar{d}, \oplus_{e \mu}\right\} \\
& \rightarrow\left\{(u, \bar{d}),(\bar{u}, d), \oplus_{e \mu},(d, \bar{d}), \oplus_{e \mu},(\bar{d}, d), \oplus_{e \mu}\right\} \\
& \rightarrow\{u, \bar{d}\}+\{\bar{u}, d\}+\left\{\oplus_{e \mu}, \oplus_{d}, \oplus_{e \mu}, \oplus_{d}, \oplus_{e \mu}\right\} \\
& \rightarrow \pi^{+}+\pi^{-}+\left\{\oplus_{e \mu}, \oplus_{d}, \oplus_{e \mu}, \oplus_{d}, \oplus_{e \mu}\right\} \\
& K_{s}+Z^{0}+Z^{0} \rightarrow\left\{d,\left(\bar{d}, \oplus_{e \mu}\right),\left(d, \oplus_{e \mu}\right), \bar{d}\right\} \\
&+\left\{u, \bar{u}, d, \bar{d}, \oplus_{e \mu}\right\}+\left\{u, \bar{u}, d, \bar{d}, \oplus_{e \mu}\right\} \\
& \rightarrow\left\{(u, \bar{d}, \bar{u}, d), \oplus_{e \mu},(u, \bar{d}, \bar{u}, d), \oplus_{e \mu},\right. \\
&\left.(d, \bar{d}), \oplus_{e \mu},(\bar{d}, d), \oplus_{e \mu}\right\} \\
& \rightarrow\{u, \bar{d}, \bar{u}, d\}+\{u, \bar{d}, \bar{u}, d\}+\left\{\oplus_{e \mu}, \oplus_{e \mu}, \oplus_{d}, \oplus_{e \mu}, \oplus_{d}, \oplus_{e \mu}\right\} \\
& \rightarrow \pi^{0}+\pi^{0}+\left\{\oplus_{e \mu}, \oplus_{e \mu}, \oplus_{d}, \oplus_{e \mu}, \oplus_{d}, \oplus_{e \mu}\right\}
\end{aligned}
$$

The $K_{l}$ decay is $K_{l} \rightarrow \pi^{+}+e^{-}+\bar{v}_{e}$.

$$
\begin{aligned}
& K_{l}+Z^{0} \rightarrow\left\{d,\left(\bar{d}, \oplus_{e \mu}\right),\left(d, \oplus_{e \mu}\right), \bar{d}\right\}+\left\{u, \bar{u}, d, \bar{d}, \oplus_{e \mu}\right\} \\
& \rightarrow\left\{(u, \bar{d}),\left(\bar{u}, d, v_{e}\right), \bar{v}_{e}, \oplus_{\mu},(d, \bar{d}), \oplus_{e \mu},(\bar{d}, d), \oplus_{e \mu}\right\} \\
& \rightarrow\{u, \bar{d}\}+\left\{\bar{u}, d, v_{e}\right\}+\left\{\bar{v}_{e}\right\}+\left\{\oplus_{\mu}, \oplus_{d}, \oplus_{e \mu}, \oplus_{d}, \oplus_{e \mu}\right\} \\
& \rightarrow \pi^{+}+e^{-}+\bar{v}_{e}+\left\{\oplus_{\mu}, \oplus_{d}, \oplus_{e \mu}, \oplus_{d}, \oplus_{e \mu}\right\}
\end{aligned}
$$

The very rare $K^{+}$decay is $K^{+} \rightarrow \pi^{+}+v+\bar{v}$ by [8]. It is connsidered that $K^{+} \rightarrow \pi^{+}+v+\bar{v}+h v$. Since the $K^{+}$consists of $\left\{\left(\pi^{+}\right), \oplus_{e \mu}\right\}$, the $\oplus_{e}$ and $\oplus_{\mu}$ corresponding to $(v+\bar{v})$ and $h v$, respectively. The pair connection of the particle and its antiparticle is regarded as an annihilation in this study.

\subsection{Identity of the Lambda particle (Description of the Lambda Decay)}

The $\Lambda^{0}$ decays are shown by the three reactions.

$$
\begin{aligned}
& \Lambda^{0} \rightarrow n+\pi^{0} \\
& \Lambda^{0} \rightarrow P+\pi^{-}
\end{aligned}
$$

and

$$
\Lambda^{0} \rightarrow P+\mu^{-}+v_{\mu}
$$

The expressions of the quarks and neutrinos as follows.

$$
\begin{aligned}
& \{u, d, s\} \rightarrow\{u, d, d\}+\{u, \bar{d}, \bar{u}, d\} \\
& \{u, d, s\} \rightarrow\{(u, d, d),(u, \bar{d})\}+\{\bar{u}, d\}
\end{aligned}
$$

and

$$
\{u, d, s\} \rightarrow\{(u, d, d),(u, \bar{d})\}+\left\{\bar{u}, d, v_{e}, \bar{v}_{e}, \bar{v}_{\mu}\right\}+v_{\mu}
$$

The expressions are changed by $\{s\}=\left\{d, \oplus_{e \mu}\right\}$ $=\left\{d, \oplus_{e}, \oplus_{\mu}\right\}$.

$$
\begin{aligned}
& \left\{u, d,\left(d, \oplus_{e \mu}\right)\right\} \rightarrow\{u, d, d\}+\{u, \bar{d}, \bar{u}, d\} \\
& \left\{u, d,\left(d, \oplus_{e \mu}\right)\right\} \rightarrow\{(u, d, d),(u, \bar{d})\}+\{\bar{u}, d\}
\end{aligned}
$$

and

$$
\begin{aligned}
& \left\{u, d,\left(d, \oplus_{e \mu}\right)\right\} \\
& \rightarrow\{(u, d, d),(u, \bar{d})\}+\left\{\bar{u}, d, v_{e}, \bar{v}_{e}, \bar{v}_{\mu}\right\}+v_{\mu}
\end{aligned}
$$

It seems that the $Z^{0}$ bosons are missing in the left sides.

$$
\begin{aligned}
& \left\{u, d\left(d, \oplus_{e \mu}\right)\right\}+\left\{u, \bar{u}, d, \bar{d}, \oplus_{e \mu}\right\} \\
& \rightarrow\{u, d, d\}+\{u, \bar{d}, \bar{u}, d\} \\
& \left\{u, d,\left(d, \oplus_{e \mu}\right)\right\}+\left\{u, \bar{u}, d, \bar{d}, \oplus_{e \mu}\right\} \\
& \rightarrow\{(u, d, d),(u, \bar{d})\}+\{\bar{u}, d\}
\end{aligned}
$$

and

$$
\begin{aligned}
& \left\{u, d,\left(d, \oplus_{e \mu}\right)\right\}+\left\{u, \bar{u}, d, \bar{d}, \oplus_{e \mu}\right\} \\
& \rightarrow\{(u, d, d),(u, \bar{d})\}\left\{\oplus_{e \mu}\right\}+\left\{\bar{u}, d, v_{e}, \bar{v}_{e}, \bar{v}_{\mu}\right\}+v_{\mu}
\end{aligned}
$$

The reactions with the addition of photon as follows. 


$$
\begin{aligned}
\Lambda^{0}+Z^{0} & \rightarrow\left\{u, d,\left(d, \oplus_{e \mu}\right)\right\}+\left\{u, \bar{u}, d, \bar{d}, \oplus_{e \mu}\right\} \\
& \rightarrow\left\{u, d,\left(d, \oplus_{e \mu}\right),\left(u, \bar{u}, d, \bar{d}, \oplus_{e \mu}\right)\right\} \\
& \rightarrow\{u, d, d\}+\{u, \bar{d}, \bar{u}, d\}+\left\{\oplus_{e \mu}\right\}\left\{\oplus_{e \mu}\right\} \\
& \rightarrow n+\pi^{0}+\left\{\oplus_{e \mu}\right\}\left\{\oplus_{e \mu}\right\} \\
\Lambda^{0}+Z^{0} \rightarrow & \left\{u, d,\left(d, \oplus_{e \mu}\right)\right\}+\left\{u, \bar{u}, d, \bar{d}, \oplus_{e \mu}\right\} \\
\rightarrow & \left\{u, d,\left(d, \oplus_{e \mu}\right),\left(u, \bar{u}, d, \bar{d}, \oplus_{e \mu}\right)\right\} \\
\rightarrow & \{(u, d, d),(u, \bar{d})\}+\{\bar{u}, d\}+\left\{\oplus_{e \mu}\right\}\left\{\oplus_{e \mu}\right\} \\
\rightarrow & P+\pi^{-}+\left\{\oplus_{e \mu}\right\}\left\{\oplus_{e \mu}\right\}
\end{aligned}
$$

and

$$
\begin{aligned}
\Lambda^{0}+Z^{0} & \rightarrow\left\{u, d,\left(d, \oplus_{e \mu}\right)\right\}+\left\{u, \bar{u}, d, \bar{d}, \oplus_{e \mu}\right\} \\
& \rightarrow\left\{u, d,\left(d, \oplus_{e \mu}\right),\left(u, \bar{u}, d, \bar{d}, v_{e}, \bar{v}_{e}, \bar{v}_{\mu}, v_{\mu}\right)\right\} \\
& \rightarrow\{(u, d, d),(u, \bar{d})\}+\left\{\left(\bar{u}, d, v_{e}\right), \bar{v}_{e}, \bar{v}_{\mu}\right\} \\
& +\left\{v_{\mu}\right\}+\left\{\oplus_{e \mu}\right\} \\
& \rightarrow P+\mu^{-}+v_{\mu}+\left\{\oplus_{e \mu}\right\}
\end{aligned}
$$

The $\Lambda^{0}$ particle consists of $\left\{u, d,\left(d, \oplus_{e \mu}\right)\right\}=$ $\left\{(u, d, d), \oplus_{e \mu}\right\}$. The $\Lambda^{0}$ particle includes the neutron.

\subsection{Identity of the Bottom and Top quark}

The $\Lambda_{b}^{0}$ consists of $(u, d, b)$. Its two decays are $\Lambda_{b}^{0}$ $\rightarrow p+\pi^{-}$and $\Lambda_{b}^{0} \rightarrow p+K^{-}$as shown by [9]. Their $\Lambda_{b}^{0}$ decays are shown.

$$
\begin{aligned}
& \{u, d, b\} \rightarrow\{(u, d, d),(u, \bar{d})\}+\{\bar{u}, d\} \\
& \{u, d, b\} \rightarrow\{(u, d, d),(u, \bar{d})\}+\left\{\bar{u}, d, \oplus_{e \mu}\right\}
\end{aligned}
$$

If $b$ quark is a composite, the minimum configuration's $b$ quark is equal to $\{d,(u, \bar{d}, \bar{u}, d)\}=\left\{d,\left(\pi^{0}\right)\right\}$. The other configuration's $b$ quark consists of

$$
\left\{d,(u, \bar{d}, \bar{u}, d),\left(\oplus_{e}, \oplus_{\mu}\right)\right\}=\left\{d,\left(\pi^{0}\right),\left(\oplus_{e \mu}\right)\right\}=\left\{d,\left(Z^{0}\right)\right\}
$$

We recognize the top and anti-top quarks produced in proton-antiproton collisions by [10]. The top quarks instantly decay into two $\mathrm{W}$ bosons and two $\mathrm{b}$ quarks. One $W$ in turn decays into a muon and a neutrino, the other into up and down quarks. The general reaction is considered.

$$
\begin{gathered}
P+\bar{P} \rightarrow t+\bar{t} \rightarrow\left(W^{+}+b\right)+\left(W^{-}+\bar{b}\right) \\
\left(W^{+}\right)=\left\{u, \bar{d}, \oplus_{e}\right\}=\left\{\left(\pi^{+}\right), \oplus_{e}\right\} \\
\left(W^{-}\right)=\left\{\bar{u}, d, \oplus_{e}\right\}=\left\{\left(\pi^{-}\right), \oplus_{e}\right\} \\
(b)=\{d,(u, \bar{d}, \bar{u}, d)\}=\left\{\bar{d},\left(\pi^{0}\right)\right\} \\
(\bar{b})=\{\bar{d},(u, \bar{d}, \bar{u}, d)\}=\left\{\bar{d},\left(\pi^{0}\right)\right\}
\end{gathered}
$$

The proton-antiproton collision, $P+\bar{P} \rightarrow(t+\bar{t}) \rightarrow$ $\left(W^{+}+b\right)+\left(W^{-}+\bar{b}\right)$, is shown from the above relationship.

$$
\begin{aligned}
P & +\bar{P}=\{(u, d, d),(u, \bar{d})\}+\{\bar{u}, \bar{d}, \bar{d}\}\{\bar{u}, d\} \\
\rightarrow & (t+\bar{t}) \rightarrow\left(W^{+}+b\right)+\left(W^{-}+\bar{b}\right) \\
= & \left\{u, \bar{d}, \oplus_{e}\right\}+\{d,(u, \bar{d}, \bar{u}, d)\} \\
& +\left\{\bar{u}, d, \oplus_{e}\right\}+\{\bar{d},(u, \bar{d}, \bar{u}, d)\} \\
= & \left\{\left(u, \bar{d}, \oplus_{e}\right), v_{e}\right\}+\{d,(u, \bar{d}, \bar{u}, d)\} \\
& +\left\{\left(\bar{u}, d, \oplus_{e}\right), \bar{v}_{e}\right\}+\{\bar{d},(u, \bar{d}, \bar{u}, d)\}
\end{aligned}
$$

The anti-proton is used by $\{(\bar{u}, \bar{d} \cdot \bar{d}),(\bar{u}, d)\}$ instead of $\{\bar{u}, \bar{u}, \bar{d}\}$. It can not explain by $\{\bar{u}, \bar{u}, \bar{d}\}$. Therefore the anti-proton is either by the $\{\bar{u}, \bar{d}, \bar{d}\} \quad\{\bar{u}, d\}$ or $\{\bar{u}, \bar{d}, \bar{d}, \bar{u}, d\}$ structure in nature. The following is the $(t+\bar{t})$ configuration.

$$
\begin{aligned}
& (t+\bar{t})=\left(W^{+}+b\right)+\left(W^{-}+\bar{b}\right) \\
& =\left\{u, \bar{d}, \oplus_{e}\right\}+\{d,(u, \bar{d}, \bar{u}, d)\}+\left\{\bar{u}, d, \oplus_{e}\right\}+\{\bar{d},(u, \bar{d}, \bar{u}, d)\} \\
& =\left\{(u, d, d),(u, \bar{d}),\left(\oplus_{e}\right), \bar{d}, \bar{u}\right\}+\left\{(\bar{u}, \bar{d}, \bar{d}),(\bar{u}, d),\left(\oplus_{e}\right),(u, d)\right\} \\
& =\{(u, d, d),(u, \bar{d})\}+\{(\bar{u}, \bar{d}, \bar{d}),(\bar{u}, d)\}+\left\{(u, \bar{d}, \bar{u}, d),\left(\oplus_{e}, \oplus_{e}\right)\right\} \\
& =\{(u, d, d),(u, \bar{d})\}+\{(\bar{u}, \bar{d}, \bar{d}),(\bar{u}, d)\}+\left\{\left(\pi^{0}\right), \oplus_{e e}\right\}
\end{aligned}
$$

Thus, the proton-antiproton collision needs the component of $\left\{\left(\pi^{0}\right), \oplus_{e e}\right\}=\left\{\left(Z^{0}\right)\right\}$. The following is the ideal expression of a series of proton-antiproton collisions. 


$$
\begin{aligned}
& P+\bar{P}+\left\{\left(\pi^{0}\right), \oplus_{e e}\right\} \rightarrow\{(u, d, d),(u, \bar{d})\}+\{(\bar{u}, \bar{d}, \bar{d}),(\bar{u}, d)\}+\left\{\left(\pi^{0}\right),\left(\oplus_{e}, \oplus_{e}\right)\right\} \\
& \rightarrow(t+\bar{t}) \rightarrow\left\{(u, d, d),(u, \bar{d}),\left(\oplus_{e}\right),(\bar{d}, \bar{u})\right\}+\left\{(\bar{u}, \bar{d}, \bar{d}),(\bar{u}, d),\left(\oplus_{e}\right),(u, d)\right\} \\
& =\left\{u,(u, d, d),(\bar{u}, \bar{d}, \bar{d}),\left(\oplus_{e}\right)\right\}+\left\{\bar{u},(u, d, d),(\bar{u}, \bar{d}, \bar{d}),\left(\oplus_{e}\right)\right\} \\
& =\left\{u,(n, \bar{n}),\left(\oplus_{e}\right)\right\}+\left\{\bar{u},(n, \bar{n}),\left(\oplus_{e}\right)\right\} \\
& \rightarrow\left(W^{+}+b\right)+\left(W^{-}+\bar{b}\right) \rightarrow\left\{u, \bar{d}, \oplus_{e}\right\}+\{d,(u, \bar{d}, \bar{u}, d)\}+\left\{\bar{u}, d, \oplus_{e}\right\}+\{\bar{d},(u, \bar{d}, \bar{u}, d)\} \\
& \rightarrow\left\{\left(u, \bar{d}, v_{e}\right), \bar{v}_{e}\right\}+\{d,(u, \bar{d}, \bar{u}, d)\}+\left\{\left(\left(\bar{u}, d, v_{e}\right), \bar{v}_{e}\right\}+\{\bar{d},(u, \bar{d}, \bar{u}, d)\}\right. \\
& \rightarrow\left(e^{+}+v_{e}+\left\{d,\left(\pi^{0}\right)\right\}\right)+\left(e^{-}+\bar{v}_{e}+\left\{\bar{d},\left(\pi^{0}\right)\right\}\right)
\end{aligned}
$$

From the above nuclear reactions, the energy quanta pair is a pair of $\oplus_{e}$ and $\oplus_{e}$, not a pair of $\oplus_{e}$ and $\oplus_{\mu}$. Therefore we try to use the $\left\{\oplus_{e}, \oplus_{\mu}\right\}$ pair instead of the $\left\{\oplus_{e}, \oplus_{e}\right\}$ one.

The expression such as the experiment is considered by the following.

$$
P+\bar{P}+\left\{\left(\pi^{0}\right), \oplus_{e \mu}\right\}
$$

or

$$
\begin{aligned}
& +\bar{P}+\left\{\left(Z^{0}\right)\right\} \\
\rightarrow & \{(u, d, d),(u, \bar{d})\}+\{(\bar{u}, \bar{d}, \bar{d}),(\bar{u}, d)\} \\
& +\left\{\left(\pi^{0}\right),\left(\oplus_{e \mu}\right)\right\} \\
\rightarrow & (t+\bar{t}) \\
\rightarrow & \{(u, d, d),(u, \bar{d}),(d, \bar{u})\} \\
& +\left\{\left((\bar{u}, \bar{d}, \bar{d}),(\bar{u}, d),\left(\oplus_{e \mu}\right),(u, d)\right\}\right. \\
\rightarrow & \left(\pi^{+}+b\right)+\left(W_{2}^{-}+\bar{b}\right) \\
\rightarrow & \{u, \bar{d}\}+\{d,(u, \bar{d}, \bar{u}, d)\} \\
& +\left\{\bar{u}, d, \oplus_{e \mu}\right\}+\{\bar{d},(u, \bar{d}, \bar{u}, d)\} \\
\rightarrow & \{u, \bar{d}\}+\{d,(u, \bar{d}, \bar{u}, d)\}+\left\{\left(\bar{u}, d, v_{e}, \bar{v}_{e}, v_{\mu}\right),\left(\bar{v}_{\mu}\right)\right\} \\
& +\{\bar{d},(u, \bar{d}, \bar{u}, d)\} \\
\rightarrow & \left(u+\bar{d}+\left\{d,\left(\pi^{0}\right)\right\}\right)+\left(\mu^{-}+\bar{v}_{\mu}+\left\{\bar{d},\left(\pi^{0}\right)\right\}\right)
\end{aligned}
$$

The $\left\{\oplus_{e}, \oplus_{\mu}\right\}$ pair may be prefered through all reactions than the symmetric experiment with the $\left\{\oplus_{e}, \oplus_{e}\right\}$ pair. Those reactions are not a two-body collision.

\subsection{Identity of Higgs Boson}

The followings show the compositions of some hypothetical Higgs bosons by $[11,12]$.
The light Higgs boson

$$
\begin{aligned}
\left(H^{0}\right)_{\text {light }} & =\{(b),(\bar{b})\} \\
& =\{(d,(u, \bar{d}, \bar{u}, d)),(\bar{d},(u, \bar{d}, \bar{u}, d))\} \\
& =\left\{\left(\pi^{0}\right), d, \bar{d},\left(\pi^{0}\right)\right\}
\end{aligned}
$$

The heavy Higgs bosons

$$
\begin{aligned}
\left(H^{0}\right)_{\text {heavy }(1)} & =\left\{\left(W^{+}\right),\left(W^{-}\right)\right\} \\
& =\left\{u, \bar{d}, \oplus_{e}\right\}\left\{\bar{u}, d, \oplus_{e}\right\} \\
& =\left\{\left(\pi^{0}\right), \oplus_{e e}\right\}
\end{aligned}
$$

and

$$
\begin{aligned}
\left(H^{0}\right)_{\text {heavy }(2)} & =\left\{\left(Z^{0}\right),\left(Z^{0}\right)\right\}=\left\{(u, \bar{u}),\left(K^{0}\right),\left(Z^{0}\right)\right\} \\
& =\left\{\left(\pi^{0}\right),\left(\pi^{0}\right), \oplus_{e \mu}, \oplus_{e \mu}\right\}
\end{aligned}
$$

A neutral pion $\left\{\left(\pi^{0}\right)\right\}$ is the common minimum particle of Higgs boson.

\subsection{Identity of Pentaquark Composite $\Theta^{+}$}

It was reported on the evidence of pentaquark composite $\left(\Theta^{+}\right)$by [13].

$$
\begin{aligned}
& \gamma+n+p+(\text { etc. }) \rightarrow(\text { composite }) \\
& \rightarrow \Theta^{+}\{u, u, d, d, \bar{s}\}+\left(K^{-}+p\right)+(\text { others }) \\
& \rightarrow\left(n+K^{+}\right)+\left(K^{-}+p\right)+(\text { others })
\end{aligned}
$$

\subsubsection{Analisys}

The composition of pentaquark composite is analized. The following is the new expression.

$$
\begin{aligned}
& \gamma+\pi^{0}+n+P+(\text { etc. }) \rightarrow \text { composite }\left\{\gamma, \pi^{0}, n, P,(\text { etc. })\right\} \\
& \rightarrow \Theta^{+}\{u, u, d, d,(\bar{s})\}+\left(\left(K^{-}+P\right)\right)+(\text { others }) \\
& \rightarrow\left(n+K^{+}\right)+\left(K^{-}+P\right)+(\text { others })
\end{aligned}
$$


Next is the simple expression.

$$
\begin{aligned}
\gamma & +\pi^{0}+n+P \\
\rightarrow & \left\{\left(\oplus_{e \mu}, \oplus_{e \mu}\right),(u, \bar{d}, \bar{u}, d),(u, d, d),((u, d, d)),(u, \bar{d})\right\} \\
\rightarrow & \Theta^{+}\left\{(u, d, d, u, \bar{d}),\left(\oplus_{e \mu}\right)\right\} \\
& +\left\{\bar{u}, d, \oplus_{e \mu}\right\}+\{(u, d, d),(u, \bar{d})\} \\
\rightarrow & \{u, d, d\}+\left\{u, \bar{d}, \oplus_{e \mu}\right\}+\left\{\bar{u}, d, \oplus_{e \mu}\right\}+\{(u, d, d),(u, \bar{d})\} \\
\rightarrow & \left(n+K^{+}\right)+\left(K^{-}+P\right)
\end{aligned}
$$

This pentaquark composite $\left(\Theta^{+}\right)$consists of the energy quanta and the $\mathrm{f}^{+}$ive members of quark. However this reaction can be also explained with the traditional proton $\{u, u, d\}$.

$$
\begin{aligned}
& \gamma+\pi^{0}+n+p \\
& \rightarrow\left\{\left(\oplus_{e \mu}, \oplus_{e \mu}\right),(u, \bar{d}, \bar{u}, d),(u, d, d),(u, u, d)\right\} \\
& \rightarrow \Theta^{+}\left\{(u, d, d, u, \bar{d}),\left(\oplus_{e \mu}\right)\right\}+\left\{\bar{u}, d, \oplus_{e \mu}\right\}+\{(u, u, d)\} \\
& \rightarrow\{u, d, d\}+\left\{u, \bar{d}, \oplus_{e \mu}\right\}+\left\{\bar{u}, d, \oplus_{e \mu}\right\}+\{(u, u, d)\} \\
& \rightarrow\left(n+K^{+}\right)+\left(K^{-}+p\right)
\end{aligned}
$$

\subsection{Identity of the Debated Pentaquark Composite $\Theta^{+}$}

\subsubsection{JLab News Release, Pentaquark Debate \\ Heats Up (April 28)}

It was reported that "Pentaquarks are built of five quarks: for example, the $\Theta^{+}$is built of two up quarks, two down quarks and an anti-strange quark." by [14].

$$
p+\gamma \rightarrow \Theta^{+}+\bar{K}^{0} \rightarrow\left(K^{+}+n\right)+\left(\pi^{+}+\pi^{-}\right)
$$

\subsubsection{Results of Analysis}

The composition of $\Theta^{+}$composite is analized. The proton decays with a neutral pion $\left\{\pi^{0}\right\}$ and some gammarays by a law of conservation of number of particle. It is shown that the $\Theta^{+}$compositions are $\{u, d, d, u, \bar{d}$, $\left.\oplus_{e \mu}\right\}$. It is an exotic composite.

$$
\begin{aligned}
& P+\gamma+\pi^{0}+\gamma \text { or } P+\gamma \\
&= P+\left\{\oplus_{e \mu}\right\}+\pi^{0}+\left\{\oplus_{e \mu}\right\} \text { or }=P+\left\{\oplus_{e \mu}\right\}+Z^{0} \\
&=\left\{(P), \oplus_{e \mu}\right\}+\left\{\left(\pi^{0}\right), \oplus_{e \mu}\right\} \\
& \rightarrow \Theta^{+}\left\{(P), \oplus_{e \mu}\right\}+\left\{\oplus_{u},\left(\bar{K}^{0}\right)\right\} \\
&\left(\rightarrow \Theta^{+}\left\{(n),\left(\pi^{+}\right), \oplus_{e \mu}\right\}+Z^{0}\right) \\
& \rightarrow \Theta^{+}\left\{\left(\pi^{+}, \oplus_{e \mu}\right),(n)\right\}+\left\{\left(\pi^{0}\right), \oplus_{e \mu}\right\} \\
& \rightarrow\left(K^{+}+n\right)+\left(\pi^{+}+\pi^{-}\right)+\left\{\oplus_{e \mu}\right\}
\end{aligned}
$$

$$
\begin{aligned}
& (n)=\{u, d, d\} \\
& (P)=\{(u, d, d),(u, \bar{d})\} \\
& \left(\pi^{+}\right)=\{u, \bar{d}\} \\
& \left(\pi^{-}\right)=\{\bar{u}, d\} \\
& \left(\pi^{0}\right)=\{u, \bar{d}, \bar{u}, d\} \\
& (\bar{s})=\left\{\bar{d}, \oplus_{e \mu}\right\} \\
& \left(K^{+}\right)=\left\{(u, \bar{d}), \oplus_{e \mu}\right\}=\left\{\left(\pi^{+}\right), \oplus_{e \mu}\right\} \\
& \left(\bar{K}^{0}\right)=\left\{(d, \bar{d}), \oplus_{e \mu}\right\}=\{d,(\bar{s})\}=\left\{d,\left(\bar{d}, \oplus_{e \mu}\right)\right\}
\end{aligned}
$$

\subsubsection{Details of Analysis}

$$
\begin{aligned}
& \gamma+P \rightarrow \Theta^{+}+\bar{K}^{0} \rightarrow\left(K^{+}+n\right)+\left(\pi^{+}+\pi^{-}\right) \\
& \rightarrow\left\{u, \bar{d}, \oplus_{e \mu}\right\}+\{u, d, d\}+\{u, \bar{d}\}+\{\bar{u}, d\} \\
& \rightarrow\left\{u, \bar{d}, \oplus_{e \mu}\right\}+\{u, d, d\}+\{u, \bar{d}\}+\{\bar{u}, d\}+\{\text { etc }\}
\end{aligned}
$$

The $\{u, \bar{d}\}+\{\bar{u}, d\}+\{e t c\}$ must have the components of $\bar{K}^{0} \quad\left(=\left\{d, \bar{d}, \oplus_{e \mu}\right\}\right)$. The $\{$ etc $\}$ is $\left\{\oplus_{e \mu}\right\}$.

$$
\begin{aligned}
& \rightarrow\left\{(u, \bar{d}), \oplus_{e \mu}\right\}+\{u, d, d\}+\{u, \bar{d}\}+\{\bar{u}, d\}+\left\{\oplus_{e \mu}\right\} \\
& \rightarrow\left\{(u, \bar{d}), \oplus_{e \mu},(u, d, d)\right\}+\left\{(u, \bar{d}),(\bar{u}, d), \oplus_{e \mu}\right\} \\
& \rightarrow\left\{(u, \bar{d}),(u, d, d), \oplus_{e \mu}\right\}+\left\{(u, \bar{d}, \bar{u}, d), \oplus_{e \mu}\right\} \\
& \rightarrow \Theta^{+}\left\{(P), \oplus_{e \mu}\right\}+\left\{\left(\pi^{0}\right), \oplus_{e \mu}\right\} \text { or } \Theta^{+}\left\{(P), \oplus_{e \mu}\right\}+Z^{0} \\
& \rightarrow P+\left\{\oplus_{e \mu}\right\}+\pi^{0}+\left\{\oplus_{e \mu}\right\} \rightarrow P+\gamma+\pi^{0}+\gamma
\end{aligned}
$$

(If $\pi^{0}$ is a kind of $\gamma$ ) $\cdots P+\gamma$

\subsubsection{Analized Description}

$$
\begin{aligned}
& \gamma+P+\pi^{0}+\gamma \\
& \{(P)\}+\left\{\oplus_{e \mu}\right\}+\left\{\left(\pi^{0}\right)\right\}+\left\{\oplus_{e \mu}\right\} \\
& \rightarrow \Theta^{+}\left(=\left\{(P), \oplus_{e \mu}\right\}\right)+\left\{\left(\pi^{0}\right), \oplus_{e \mu}\right\} \\
& \text { or }=\left\{(n),\left(\pi^{+}\right), \oplus_{e \mu}\right\}+\left\{\left(Z^{0}\right)\right\} \\
& \ldots=\left\{(n),\left(K^{+}\right)\right\}+\left\{\left(Z^{0}\right)\right\} \\
& \ldots=\left\{\left(\Lambda^{0}\right)\left(\pi^{+}\right)\right\}+\left\{\left(Z^{0}\right)\right\} \\
& \rightarrow \Theta^{+}\left\{(P), \oplus_{e \mu}\right\}+\left\{(u, \bar{u}),\left(\bar{K}^{0}\right)\right\} \\
& \rightarrow\left\{(u, d, d),(u, \bar{d}), \oplus_{e \mu}\right\}+\left\{u, \bar{d}, \bar{u}, d, \oplus_{e \mu}\right\} \\
& \rightarrow\left\{(u, \bar{d}), \oplus_{e \mu},(u, d, d)\right\}+\left\{(u, \bar{d}),(\bar{u}, d), \oplus_{e \mu}\right\} \\
& \rightarrow\left\{(u, \bar{d}), \oplus_{e \mu}\right\}+\{u, d, d\}+\{u, \bar{d}\}+\{\bar{u}, d\}+\left\{\oplus_{e \mu}\right\}
\end{aligned}
$$


The $\Theta^{+}$composite consists of the newly defined proton and the energy quanta. This reaction cannot be explained with the traditional proton $\{u, u, d\}$.

\subsection{Identity of the B Composites $\left(B / B^{-}\right)$}

\subsubsection{On "Enhanced CP Violation with}

$\boldsymbol{B} \rightarrow \boldsymbol{K} \boldsymbol{D}^{0}\left(\overline{\boldsymbol{D}}^{0}\right)$ Modes and Extraction of the

Cabibbo-Kobayashi-Maskawa Angle $\gamma$ [15]"

The $B$ decay and the quark expression are as follows.

$$
\begin{aligned}
& B \rightarrow\left\{\left(\bar{D}^{0}\right)\right\}+\left\{\left(\pi^{0}\right)\right\} \\
& \rightarrow\left\{\left(\bar{D}^{0}\right)\right\}+\left\{\left(K^{+}\right)\right\}+\left\{\left(\pi^{-}\right),\left(\pi^{+}\right),\left(\pi^{-}\right)\right\}\{b, \bar{b}, u, \bar{u}\} \\
& \rightarrow\{\bar{c}, u\}+\{u, \bar{u}, d, \bar{d}\} \rightarrow\{\bar{c}, u\}+\left\{u, \bar{d}, \oplus_{e \mu}\right\} \\
& +\left\{\left(\pi^{-}\right),\left(\pi^{+}\right),\left(\pi^{-}\right)\right\}\left\{\left(d, \pi^{0}\right), \bar{u},\left(\bar{d}, \pi^{0}\right), u\right\} \\
& \rightarrow\left\{\bar{d}, \oplus_{e \mu}, \bar{u}, d, u\right\}+\left\{u, \bar{d}, \oplus_{e \mu}\right\}+\left\{\left(\pi^{-}\right),\left(\pi^{+}\right),\left(\pi^{-}\right)\right\}
\end{aligned}
$$

The undetected energies are missing. Next expression is complete.

$$
\begin{aligned}
& \left\{\left(d, \pi^{0}\right), \bar{u},\left(\bar{d}, \pi^{0}\right), u\right\}+\oplus_{e \mu}+\oplus_{e \mu} \\
& \rightarrow\left\{\bar{d}, \oplus_{e \mu}, \bar{u}, d, u\right\}+\left\{u, \bar{d}, \oplus_{e \mu}\right\} \\
& +\left\{\left(\pi^{-}\right),\left(\pi^{+}\right),\left(\pi^{-}\right)\right\}\{b, \bar{b}, u, \bar{u}\}+\oplus_{e \mu}+\oplus_{e \mu} \\
& \rightarrow\{\bar{c}, u\}+\oplus_{e \mu}+\{u, \bar{u}, d, \bar{d}\}+\{u, \bar{u}, d, \bar{d}\} \\
& \rightarrow\{\bar{c}, u\}+\left\{u, \bar{d}, \oplus_{e \mu}\right\}+\left\{\left(\pi^{-}\right),\left(\pi^{+}\right),\left(\pi^{-}\right)\right\} \\
& B+\oplus_{e \mu}+\oplus_{e \mu} \rightarrow\left\{\left(\bar{D}^{0}\right)\right\}+\oplus_{e \mu}+\left\{\pi^{0}\right\}+\left\{\pi^{0}\right\} \\
& \rightarrow\left\{\left(\bar{D}^{0}\right)+\left(\left(K^{+}\right)\right\}+\left\{\left(\pi^{-}\right),\left(\pi^{+}\right),\left(\pi^{-}\right)\right\}\right. \\
& (B)=\left\{\left(d, \pi^{0}\right), \bar{u},\left(\bar{d}, \pi^{0}\right), u\right\}=\left\{\left(\pi^{0}\right),\left(\pi^{0}\right),\left(\pi^{0}\right)\right\} \\
& \left(K^{+}\right)=\left\{(u, \bar{d}), \oplus_{e \mu}\right\}=\left\{\left(\pi^{+}\right), \oplus_{e \mu}\right\} \\
& \left(\bar{D}^{0}\right)=\{(\bar{c}), u\} r e f 16=\{(\bar{s}, \bar{u}, d), u\} \\
& \quad=\left\{\left(\bar{d}, \oplus_{e \mu}, \bar{u}, d\right), u\right\}=\{(c), \bar{u}\}=\left(D^{0}\right) \\
& (b)=\left\{d,\left(\pi^{0}\right)\right\}=\{d,((u, \bar{u}, d, \bar{d})\} \\
& (\bar{b})=\left\{\bar{d},\left(\pi^{0}\right)\right\}=\{\bar{d},(u, \bar{u}, d, \bar{d})\} \\
& (c)=\left\{(s),\left(\pi^{+}\right)\right\}=\{(s),(u, \bar{d})\} \\
& (\bar{c})=\left\{\bar{s},\left(\pi^{-}\right)\right\}=\{\bar{s},((\bar{u}, d)\} \\
& (s)=\left\{d, \oplus_{e \mu}\right\} \quad(\bar{s})=\left\{\bar{d}, \oplus_{e \mu}\right\}
\end{aligned}
$$

4.8.2. On "First Evidence of the Decay $B^{-} \rightarrow D K^{-}$ Followed by $D \rightarrow K^{+} \& \pi^{-}$(2010)"

Two $B^{-}$decays and the quark expressions are as follows [17].

(First)

$$
\begin{aligned}
& \left\{\left(B^{-}\right)\right\} \rightarrow\left\{\left(D^{0}\right)\right\}+\left\{\left(W^{-}\right)\right\} \rightarrow\left\{\left(D^{0}\right)\right\}+\left\{\left(K^{-}\right)\right\} \\
& \{(b)), \bar{u}\} \rightarrow\{(c), \bar{u}\}+\left\{\bar{u}, d, \oplus_{e}\right\} \rightarrow\{(c), \bar{u}\}+\left\{\bar{u}, d, \oplus_{e \mu}\right\} \\
& \left\{d, \pi^{0}, \bar{u}\right\} \rightarrow\left\{d, \oplus_{e \mu}, u, \bar{d}, \bar{u}\right\}+\left\{\bar{u}, d, \oplus_{e \mu}\right\}
\end{aligned}
$$

The quark expression is uncomplete. It seems that the undetected energy is missing. Next expression is complete.

$$
\begin{aligned}
\left\{d,\left(\pi^{0}\right), \bar{u}, \oplus_{e \mu}, \oplus_{e \mu}\right\} & \rightarrow\left\{d, \oplus_{e \mu}, u, \bar{d}, \bar{u}\right\}+\left\{\oplus_{\mu}\right\}+\left\{\bar{u}, d, \oplus_{e}\right\} \\
\rightarrow\left\{d, \oplus_{e \mu}, u, \bar{d}, \bar{u}\right\} & +\left\{\bar{u}, d, \oplus_{e \mu}\right\} \\
\left\{\left(B^{-}\right)\right\}+\left\{\oplus_{e \mu}\right\}+\left\{\oplus_{e \mu}\right\} & \rightarrow\left\{\left(D^{0}\right)\right\}+\left\{\oplus_{\mu}\right\}+\left\{\left(W^{-}\right)\right\} \\
& \rightarrow\left\{\left(D^{0}\right)\right\}+\left\{\left(K^{-}\right)\right\}
\end{aligned}
$$

(Second)

$$
\begin{aligned}
\left\{\left(\left(B^{-}\right)\right)\right\} & \rightarrow\{u\}+\left\{\left(\left(W^{-}\right)\right)\right\}+\{\bar{u}\} \\
\rightarrow & \left\{\left(D^{0}\right)\right\}+\left\{\left(K^{-}\right)\right\} \\
\{(b), \bar{u}\} & \rightarrow\{u\}+\left\{\bar{u}, d, \oplus_{e}\right\}+\{\bar{u}\} \\
\rightarrow & \{(\bar{c}), u\}+\{(s), \bar{u}\} \\
\left\{d,\left(\pi^{0}\right), \bar{u}\right\} & \rightarrow\{u\}+\left\{\bar{u}, d, \oplus_{e}\right\}+\{\bar{u}\} \\
& \rightarrow\left\{\left(\bar{d}, \oplus_{e \mu}, \bar{u}, d\right), u\right\}+\left\{d, \oplus_{e \mu}, \bar{u}\right\}
\end{aligned}
$$

The undetected energies and $\{d, \bar{d}\}$ are missing. Next expression is complete.

$$
\begin{aligned}
& \left\{d,\left(\pi^{0}\right), \bar{u}\right\}+\left\{\oplus_{e \mu}\right\}+\left\{\oplus_{e \mu}\right\} \\
& \rightarrow\{u\}+\{d, \bar{d}\}+\left\{\oplus_{e \mu}\right\}+\left\{\oplus_{\mu}\right\}+\left\{\bar{u}, d, \oplus_{e}\right\}+\{\bar{u}\} \\
& \rightarrow\left\{\left(\bar{d}, \oplus_{e \mu}, \bar{u}, d\right), u\right\}+\left\{d, \oplus_{e \mu}, \bar{u}\right\} \\
& \{(b), \bar{u}\}+\left\{\oplus_{e \mu}\right\}+\left\{\oplus_{e \mu}\right\} \\
& \rightarrow\{u\}+\{d, \bar{d}\}+\left\{\oplus_{e \mu}\right\}+\left\{\oplus_{\mu}\right\}+\left\{\bar{u}, d, \oplus_{e}\right\}+\{\bar{u}\} \\
& \rightarrow\{\bar{c}, u\}+\{(s), \bar{u}\} \\
& \left\{\left(B^{-}\right)\right\}+\left\{\oplus_{e \mu}\right\}+\left\{\oplus_{e \mu}\right\} \\
& \rightarrow\{u\}+\{d, \bar{d}\}+\left\{\oplus_{e \mu}\right\}+\left\{\oplus_{\mu}\right\}+\left\{\left(W^{-}\right)\right\}+\{\bar{u}\} \\
& \rightarrow\left\{\left(D^{0}\right)\right\}+\left\{\left(K^{-}\right)\right\}
\end{aligned}
$$




$$
\left(B^{-}\right)=\left\{d,\left(\pi^{0}\right), \bar{u}\right\}=\left\{\left(\pi^{-}\right),\left(\pi^{0}\right)\right\}
$$

\section{Summary}

The fundamental particles are expected to be up quark $\{u\}$, down quark $\{d\}$, neutrino $\left\{v_{e}\right\}$, muon-neutrino $\left\{v_{\mu}\right\}$, and those anti-particles. The difference between neutrino and muon-neutrino is their kinetic energies.

The leptons of electron, positron, and energy quanta have the subatomic structure.

$$
\begin{aligned}
& \left(e^{+}\right)=\left\{u, \bar{d}, \bar{v}_{e}\right\} \\
& \left(e^{-}\right)=\left\{\bar{u}, d, v_{e}\right\} \\
& \oplus_{u}=\{u, \bar{u}\} \\
& \oplus_{d}=\{d, \bar{d}\} \\
& \oplus_{e}=\left\{v_{e}, \bar{v}_{e}\right\} \\
& \oplus_{\mu}=\left\{v_{\mu}, \bar{v}_{\mu}\right\}
\end{aligned}
$$

The members of charged muons, $\mu^{+} / \mu^{-}$, were shown.

$$
\begin{aligned}
& \left(\mu^{+}\right)=\left\{u, \bar{d}, v_{e}, \bar{v}_{e} \bar{v}_{\mu}\right\}=\left\{\left(\pi^{+}\right)\right\}\left\{\oplus_{e}\right\}\left\{\bar{v}_{\mu}\right\} \\
& \left(\mu^{-}\right)=\left\{\bar{u}, d, v_{e}, \bar{v}_{e}\right\}, v_{\mu}=\left\{\left(\pi^{-}\right)\right\}\left\{\oplus_{e}\right\}\left\{v_{\mu}\right\}
\end{aligned}
$$

The members of $W^{+} / W^{-} / Z^{0}$ bosons and the minimum member of Higgs boson were shown.

$$
\begin{aligned}
& \left(W^{+}\right)=\left\{u, \bar{d}, v_{e}, \bar{v}_{e}\right\}=\left\{\left(\pi^{+}\right)\right\}\left\{\oplus_{e}\right\} \\
& \left(W^{-}\right)=\left\{\bar{u}, d, v_{e}, \bar{v}_{e}\right\}=\left\{\left(\pi^{-}\right)\right\}\left\{\oplus_{e}\right\} \\
& \left(Z^{0}\right)_{1}=\left\{u, \bar{u}, d, \bar{d}, v_{e}, \bar{v}_{e}, v_{\mu}, \bar{v}_{\mu}\right\}=\left\{\left(\pi^{0}\right)\right\}\left\{\oplus_{e \mu}\right\} \\
& \left(Z^{0}\right)_{2}=\left\{u, \bar{u}, d, \bar{d}, v_{e}, \bar{v}_{e}, v_{e}, \bar{v}_{e}\right\}=\left\{\left(\pi^{0}\right)\right\}\left\{\oplus_{e e}\right\} \\
& \text { minimum }\left(H^{0}\right)=\{u, \bar{u}, d, \bar{d}\}=\left\{\left(\pi^{0}\right)\right\}
\end{aligned}
$$

The members of $K^{+} / K^{-} / K^{0} / K_{s} / \Lambda^{0} / \Theta^{+} / B / B^{-}$ composites were shown.

$$
\begin{aligned}
\left(K^{+}\right) & =\{u,(\bar{s})\}=\left\{u,\left(\bar{d}, \oplus_{e \mu}\right)\right\} \\
& =\left\{(u, \bar{d}), \oplus_{e \mu}\right\}=\left\{\left(\pi^{+}\right), \oplus_{e \mu}\right\} \\
\left(K^{-}\right) & =\{\bar{u},(s)\}=\left\{\bar{u},\left(d, \oplus_{e \mu}\right)\right\} \\
& =\left\{(\bar{u}, d), \oplus_{e \mu}\right\}=\left\{\left(\pi^{-}\right), \oplus_{e \mu}\right\} \\
\left(K^{0}\right) & =\{d,(\bar{s})\}=\left\{d, \bar{d}, \oplus_{e \mu}\right\}=\left\{\oplus_{d}, \oplus_{e \mu}\right\} \\
\left(\bar{K}^{0}\right) & =\{\bar{d},(s)\}=\left\{\bar{d}, d, \oplus_{e \mu}\right\}=\left\{\oplus_{d}, \oplus_{e \mu}\right\}=\left(K^{0}\right) \\
\left(K_{s}\right) & =\{d,(\bar{s}),(s), \bar{d}\}=\left\{d,\left(\bar{d}, \oplus_{e \mu}\right),\left(d, \oplus_{e \mu}\right), \bar{d}\right\}
\end{aligned}
$$

$$
\begin{aligned}
\left(\Lambda^{0}\right) & =\left\{u, d,\left(d, \oplus_{e \mu}\right)\right\} \\
& =\left\{(u, d, d),\left(\oplus_{e \mu}\right)\right\}=\left\{(n),\left(\oplus_{e \mu}\right)\right\} \\
\left(\Lambda_{b}^{0}\right) & =\{(u, d, d),(u, \bar{u}, d, \bar{d})\}=\left\{(n),\left(\pi^{0}\right)\right\} \\
\left(\Theta^{+}\right) & =\left\{(u, d, d, u, \bar{d}),\left(\oplus_{e \mu}\right)\right\}=\left\{(P),\left(\oplus_{e \mu}\right)\right\} \\
(B) & =\left\{\left(d, \pi^{0}\right), \bar{u},\left(\bar{d}, \pi^{0}\right), u\right\}=\left\{\left(\pi^{0}\right),\left(\pi^{0}\right),\left(\pi^{0}\right)\right\} \\
\left(B^{-}\right) & =\left\{d,\left(\pi^{0}\right), \bar{u}\right\}=\left\{\left(\pi^{-}\right),\left(\pi^{0}\right)\right\}
\end{aligned}
$$

The second and third quark no longer say quark. They are the exotic second and third states of the first generation's quark composites.

$$
\begin{aligned}
(s) & =\left\{d, v_{e}, \bar{v}_{e}, v_{\mu}, \bar{v}_{\mu}\right\}=\left\{d, \oplus_{e \mu}\right\} \\
(c) & =\{(s),(u, \bar{d})\}=\left\{(s),\left(\pi^{+}\right)\right\} \\
& =\left\{\left(d, \oplus_{e \mu}\right),(u, \bar{d})\right\}=\left\{\left(d, \oplus_{e \mu}\right),\left(\pi^{+}\right)\right\}=\left\{d,\left(K^{+}\right)\right\} \\
& =\left\{u,\left(d, \bar{d}, \oplus_{e \mu}\right)\right\}=\left\{u,\left(\oplus_{d}, \oplus_{e \mu}\right)\right\}=\left\{u,\left(K^{0}\right)\right\} \\
(b) & =\{d,(u, \bar{d}, \bar{u}, d)\}=\left\{d,\left(\pi^{0}\right)\right\} \\
& \{(t),(\bar{t})\}_{1} \\
& =\left\{(u, d, d),(u, \bar{d}),(\bar{u}, \bar{d}, \bar{d}),(\bar{u}, d),\left(\pi^{0}\right),\left(\oplus_{e e}\right)\right\} \\
& \{(t),(\bar{t})\}_{2} \\
& =\left\{(u, d, d),(u, \bar{d}),(\bar{u}, \bar{d}, \bar{d}),(\bar{u}, d),\left(\pi^{0}\right),\left(\oplus_{e \mu}\right)\right\}
\end{aligned}
$$

The proton might be a pentaquark.

$$
\begin{aligned}
(P) & =\{(u, u, d),(d, \bar{d})\} \\
& \rightleftharpoons\{(u, d, d, u, \bar{d})\} \\
& \rightleftharpoons\{(u, d, d),(u, \bar{d})\}
\end{aligned}
$$

\section{Acknowledgement}

I thank you for great accomplishment for mankind. May peace be upon all living things forever and ever.

I thank my dead father for this study. All the inheritance from him helped me.

\section{References}

[1] Ryden, B. (2003) Introduction to Cosmology, 1st edition, Japanese language edition, (Pearson Education, Inc.), Chapter 10.

[2] M. Gell-Mann, "A Schematic Model of Baryons and Mesons," Physics Letters Vol 8, 1964, PP. 214-215.

[3] Zweig, G. (1964) “AN $\mathrm{S} U_{3}$ MODEL FOR STRONG 
INTERACTION SYMMETRY AND ITS BREAKING I," CERN Reports 8182/TH.401 and "AN SU MODEL FOR STRONG INTERACTION SYMMETRY AND ITS BREAKING II 8419/TH.412"

[4] Hamatsu, R. (1990) High Energy Physics of physics lecture course, Tokyo Metropolitan University.

[5] A. Kernan, W. M. Powell, C. L. Sandler, W.L. Knight, and F. Russell Stannard, "Muonic-Decay Branching Ratio of the Lambda Hyperon," Phys. Rev. Vol 133, 1964, B1271-B1273.

[6] Yuksel, H, (2006) "Positron Annihilations at the Galactic Center: Generating More Questions Than Answers," arXiv:astro-ph/0609139v1.

[7] W. M. Yao et al. (Particle Data Group), "Review of Particle Physics," J. Phys. G Vol 33, 2006, PP. 1-1232. and 2007 partial update for edition 2008, http://pdg.lbl.gov/

[8] A. F. Falk, A Lewandowski, A.A. Petrov, "Effects from the charm scale in $K^{+} \rightarrow \pi+v^{-} v$," Phys. Lett. B Vol 505, 2001, PP. 107-112.

[9] J.-E. Augustin et al., "Discovery of a Narrow Resonance in $\mathrm{e}^{+} e^{-}$Annihilation," Phys. Rev. Lett. Vol 33, 1974, PP. 1406-1408.

[10] F. Abe et al., "Identification of top quarks using kinematic variables," Phys. Rev. D Vol 52, 1995, PP. R2605-R2609.

[11] P. Igo-Kemenes, "Searches for Higgs bosons at LEP2," J. Phys. G: Nucl. Part. Phys. Vol 24, 1998, PP. 325-335 and Updated October 2005 by P. Igo-Kemenes.

[12] P.W. Higgs, "Broken Symmetries and the Masses of Gauge Bosons," Phys. Rev. Lett. Vol 13, 1964, PP. 508-509.

[13] T. Nakano et al., "Evidence for a Narrow $\mathrm{S}=+1$ Baryon Resonance in Photoproduction from the Neutron," Phys. Rev. Lett. Vol 91, 2003, PP. 012002.

[14] JLAB News Release, (2005) Pentaquark debate heats up, http://www.jlab.org/divdept/diro/publicaairs/newsreleases /2005/pentaquarks.html

[15] D. Atwood, I. Dunietz, and A. Soni, "Enhanced CP Violation with $\mathrm{B} \rightarrow K D^{0}\left(\bar{D}^{0}\right)$ Modes and Extraction of the Cabibbo-Kobayashi-Maskawa Angle $\gamma$," Phys. Rev. Lett. Vol 78, 1997, PP. 3257-3260.

[16] S. Eidelman, et al., "Charm Dalitz Plot Analysis Formalism and Results," Phys. Lett. B Vol 592, 2004, PP. 1-1109.

[17] Horii, Y, et al., (2010) First Evidence of the Decay $\mathrm{B}^{-} \rightarrow D K^{-}$Followed by $\mathrm{D} \rightarrow K^{+} \& \pi^{-}$, http://belle.kek.jp/results/summer10/dk_ads/. 